\title{
Development of the Scanning Atom Probe
}

\author{
A. Cerezo \\ Department of Materials, University of Oxford, Parks Road, Oxford OX1 3PH, U.K.
}

In 1994, Nishikawa proposed a new design of atom probe instrument, which he called the scanning atom probe (SAP) [1]. The aim of this instrument was to allow the use of specimens in the form of microtips on a planar substrate, and thus extend the applicability of atom probe microanalysis to surfaces and ultra-thin film materials. Fig. 1 shows a schematic of the design of the scanning atom probe. A microelectrode is used to raise the local electric field above one of the microtips to the level required for field evaporation of atoms at the specimen surface, and hence permit atomic-scale analysis of the apex region. Without the electrode, the presence of the flat substrate would limit the field at the apex and prevent removal of atoms. Since the presence of this microelectrode close to the specimen is a critical part of the design, the alternative naming of local electrode atom probe (LEAP) has been used by Kelly [2]. The electrode itself is mounted on a suitably fine movement XYZ stage, driven by piezoelectric motors, for example, allowing it to be aligned above a specific microtip with sub-micron accuracy. Moving the microelectrode across the surface allows individual microtips to be addressed and analysed in turn.

Microtip specimens can in principle be fabricated from thin films or devices on a planar substrate, either by masking and ion-beam milling [3, 4], or by lithography and focussed ionbeam milling [5] or by a combination of the two methods. Fig 2. shows an example of a microtip made in a silicon substrate though masking with a diamond particle and ion milling with argon ions. These methods would allow many hundreds of microtips to be made within a few $\mathrm{mm}^{2}$ of specimen, all with the layers of interest at the apex, thus greatly increasing the volume of information accessible by the atom probe. In addition, the SAP could be used to chemically analyse protrusions on surfaces without any additional specimen preparation. Fig. 3. shows a mass spectrum from the surface of a diamond field emitter which has not undergone any special treatment before analysis in the SAP [6].

Apart from extending the range of specimens that can be analysed in the atom probe, the use of a local electrode reduces by up to a factor of 3 the voltage needed to produce field evaporation [7]. This allows the analysis of specimens with a larger radius of curvature, which is useful where fabrication of specimens is difficult but also gives an increased field of view, as the magnification in the atom probe is inversely proportional to the apex radius. Reduction in the pulse voltage required allows an increase in the pulsing frequency, which in turn yield an increase in the data acquisition rate for the instrument. Currently the typical time for analysis of a volume $20 \mathrm{~nm} \times 20 \mathrm{~nm} \times 100 \mathrm{~nm}$ is many hours, so increasing the data acquisition rate is clearly necessary to permit larger volumes of material to be studied from a single specimen. The use of a local electrode has the additional advantage that it should be possible to increase the mass resolution of the instrument beyond that normally seen in a straight flight path atom probe but without the complexity of electrostatic lenses. Given a fixed energy spread of field evaporated ions (typically 1\%) post-acceleration can be used to reduce the relative energy spread over the flight path [2]. Alternatively, a dual microelectrode arrangement, as shown in Fig. 4. can be used to accelerate and decelerate the ions within the peak of the evaporation pulse, such that the ions have a fixed energy given by the applied d.c. voltage [8]. 


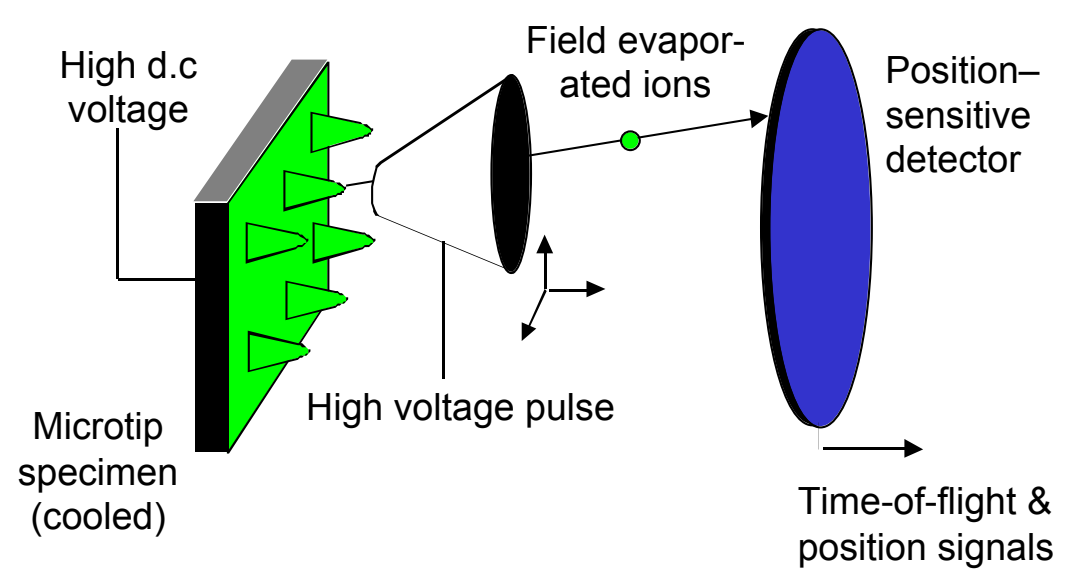

FIG. 1. Schematic diagram of a scanning atom probe instrument.

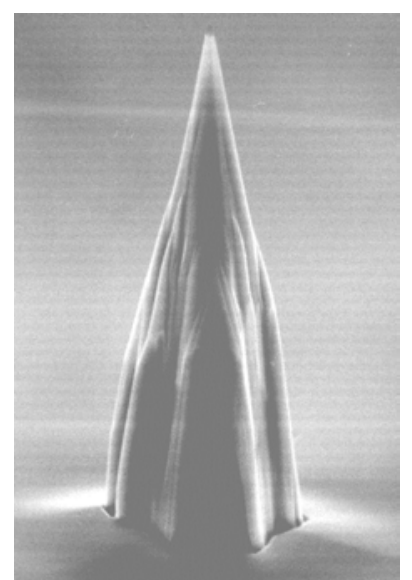

FIG. 2. Example of a silicon microtip sample made by masked ion milling

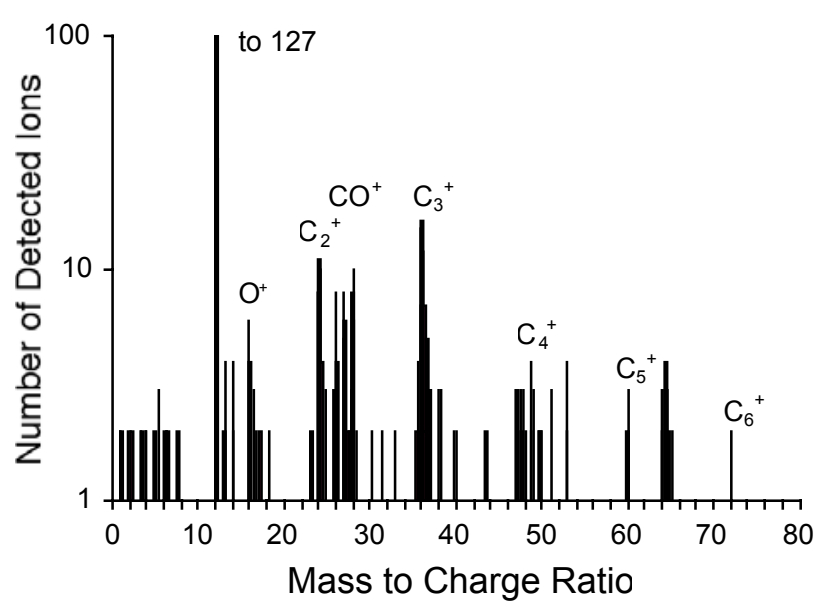

FIG. 3. Mass spectrum from the SAP analysis of the surface of a diamond field emitter [5].

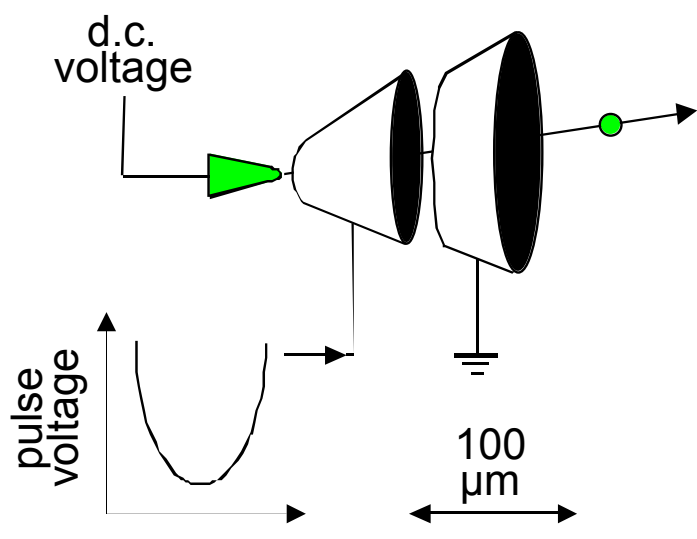

FIG. 4. Schematic of dual microelectrode arrangement for increasing mass resolution in the scanning atom probe [8].

\section{References}

[1] O. Nishikawa and M. Kimoto, Appl. Surf. Sci. 76/77 (1994) 424.

[2] T.F. Kelly et al., Ultramicroscopy 62 (1995) 29.

[3] J. A. Liddle et al., J. Phys. (Paris) Colloq. 49-C6 (1988) 509.

[4] D. J. Larson et al., Appl. Surf. Sci. 87/88 (1995) 446.

[5] D. J. Larson et al., J. Appl. Phys. 87 (2000), 5989.

[6] O. Nishikawa et al., J. Vac. Sci. Technol. B 18 (2000) 653.

[7] M. Huang et al., Ultramicroscopy 89 (2001) 163.

[8] A. Cerezo et al., Rev. Sci. Instrum. 71 (2000) 3016.

Acknowledgements

I would like to acknowledge Dr. Min Huang for his work on the masked ion milling of SAP specimens. Fig. 3 is reproduced courtesy of Prof. O. Nishikawa and J. Vac. Sci. Technol. B. 